\title{
Long Noncoding RNA LINCOI4I0 Suppresses Tumorigenesis and Enhances Radiosensitivity in Neuroblastoma Cells Through Regulating miR-545-3p/HK2 Axis
}

\author{
Liping Mou' \\ Lili Wang ${ }^{2}$ \\ Shaoming Zhang ${ }^{3}$ \\ Qinghua Wang ${ }^{4}$ \\ 'Department of Child Healthcare, People's \\ Hospital of Rizhao, Rizhao, 276800, \\ Shandong, People's Republic of China; \\ ${ }^{2}$ Department of Pediatrics, People's \\ Hospital of Rizhao, Rizhao, 276800, \\ Shandong, People's Republic of China; \\ ${ }^{3}$ Department of Neonatology, People's \\ Hospital of Rizhao, Rizhao, 276800 , \\ Shandong, People's Republic of China; \\ ${ }^{4}$ Department of Laboratory, People's \\ Hospital of Rizhao, Rizhao, 276800, \\ Shandong, People's Republic of China
}

Correspondence: Qinghua Wang Department of Laboratory, People's Hospital of Rizhao, No. 126, Tai'an Road, Rizhao, 276800, Shandong, People's

Republic of China

Tel +86-633-3365100

Email Iwnl5ya@I63.com
Background: Abnormal expression of long noncoding RNAs (lncRNAs) was often involved in tumorigenesis and radiosensitivity of various cancers. The aim of this study was to explore the biological function and regulatory mechanism of lncRNA long intergenic non-protein coding RNA 1410 (LINC01410) in tumorigenesis and radiosensitivity of neuroblastoma (NB).

Methods: The expression of LINC01410, microRNA-329-3p (miR-545-3p) and hexokinase 2 (HK2) was detected by quantitative real-time polymerase chain reaction (qRT-PCR). Methylthiazolyldiphenyl tetrazolium bromide (MTT) assay, colony formation assay and transwell assay were utilized to detect cell viability, colony formation and cell invasion abilities. Glucose consumption or lactate production was measured by glucose assay kit or lactate assay kit, respectively. The interaction between miR-545-3p and LINC01410 or HK2 was predicted by starBase v2.0 and verified by dual-luciferase reporter, RNA Immunoprecipitation (RIP) and RNA pull-down assays. Western blot was used to measure the protein expression of HK2. The mice xenograft model was established to investigate the role of LINC01410 in vivo.

Results: LINC01410 and HK2 were highly expressed while miR-545-3p was lowly expressed in NB tissues and cells. LINC01410 knockdown inhibited tumorigenesis by repressing cell proliferation and invasion, and increased the radiosensitivity via inhibiting colony formation rates and glycolysis. LINC01410 knockdown also suppressed tumor growth in vivo. Moreover, miR-545-3p could bind to LINC01410 and its downregulation reversed the effects of LINC01410 knockdown on tumorigenesis and radiosensitivity. Additionally, HK2 was a direct target of miR-545-3p and its overexpression attenuated the effects of miR-545-3p restoration on suppression of tumorigenesis and promotion of radiosensitivity. Besides, LINC01410 functioned as a molecular sponge of miR-545-3p to regulate HK2 expression.

Conclusion: LINC01410 interference inhibited tumorigenesis and increased radiosensitivity via regulating miR-545-3p/HK2 axis, providing a novel therapeutic strategy for NB.

Keywords: NB, LINC01410, miR-545-3p, HK2, tumorigenesis, radiosensitivity

\section{Introduction}

Neuroblastoma (NB) is one of the most frequent extracranial childhood tumors and originates from primitive neural crest cells of the sympathetic nervous system, accounting for $7 \%$ of malignant tumors in children and approximately $15 \%$ of all childhood cancer deaths. ${ }^{1,2}$ In spite of advances in treatment have improved the 
survival times of NB patients, children with regional or distant metastatic disease still have a poor prognosis. ${ }^{3}$ Radiotherapy is the main treatment for tumors, but the efficacy of radiotherapy in treating NB is limited due to obtaining radioresistance in the treatment of this disease. ${ }^{4}$ Thus, it is necessary to explain the molecular mechanisms of NB and identify more effective therapeutic targets to increase the radiosensitivity.

Long non-coding RNAs (lncRNAs), highly conserved transcripts ( $>200$ nucleotides), lack protein-coding capacity and play regulatory roles in various physiopathology processes. ${ }^{5}$ Currently, numerous studies have demonstrated that lncRNAs are abnormally expressed in a variety of cancers, including NB. ${ }^{6}$ For instance, lncRNA NBAT-1 downregulation promoted aggressive $\mathrm{NB}$ through enhancing proliferation and attenuating differentiation of neuronal precursors. ${ }^{7}$ Moreover, lncRNA RMRP was overexpressed in NB tissues and its knockdown suppressed the progression of NB. ${ }^{8}$ Importantly, recent researches have proven that lncRNA long intergenic non-protein coding RNA 1410 (LINC01410) expression is tightly linked to the progression of multiple cancers, such as thyroid cancer, ${ }^{9}$ cervical cancer, ${ }^{10}$ and gastric cancer. ${ }^{11}$ Besides, pervious study uncovered that LINC01410 was highly expressed in NB tissues and its expression was related to NB development. ${ }^{12}$ However, the underlying mechanisms of LINC01410 involved in regulating the progression of $\mathrm{NB}$ and radiosensitivity remain largely unknown.

Increasing evidence reveals that IncRNAs serve as competing endogenous RNAs (ceRNAs) to modulate gene expression through sponging microRNA (miRNA). ${ }^{13}$ MiRNAs are usually small RNAs (18-25 nucleotides) that lack proteincoding potential and negatively gene expression via combining with complementary sequences on target mRNA. ${ }^{14,15}$ Currently, accumulating evidence demonstrated that aberrant expression miRNA was closely related to the development and progression of cancers, and could affect the cells response to radiation. ${ }^{16,17}$ MiR-545-3p has been suggested to function as a tumor suppressor or tumor promoter in different cancers. ${ }^{18,19}$ Moreover, miR-545 was also reported to be lowly expressed in $\mathrm{NB} .{ }^{20}$ Nevertheless, the functions of miR-545-3p on the tumorigenesis and radiosensitivity of $\mathrm{NB}$ are poorly understood.

Several miRNAs target hexokinase 2 (HK2; a metabolism-related factor) to influence the progression of diverse types of cancer. ${ }^{21,22} \mathrm{HK} 2$ has been reported to be upregulated in NB and acts as a target of miR-143-3p in $\mathrm{NB}^{23}$ However, there is no report on the interaction between miR-545-3p and HK2, and exact roles of HK2 in NB progression and radiosensitivity should be explored.

In the present study, the expression of LINC01410, miR545-3p and HK2 was analyzed in NB tissues and cells. In addition, we explored their effects on proliferation, invasion, glycolysis, and radiosensitivity, and investigated the regulatory network of LINC01410/miR-545-3p/HK2 in NB cells, aiming to offer a new biological target for treatment of NB.

\section{Materials and Methods}

\section{Clinical Tissue Samples}

In this study, thirty NB tissues and paired normal tissues were obtained from patients undergoing surgery at People's Hospital of Rizhao. The patients did not receive any treatment before surgery. Each patient has signed written informed consent. This research was permitted by Research Ethics Committee of People's Hospital of Rizhao and performed in accordance with the Declaration of Helsinki. Excised tissues were collected and promptly frozen in liquid nitrogen, and then cryopreserved at $-80^{\circ}$ C for subsequent study.

\section{Cell Culture and Transfection}

Human embryonic kidney (HEK293) and NB cell line (SK-N-BE(2)) were bought from $\mathrm{BeNa}$ Culture Collection (Beijing, China). The NB cell line (GI-LI-N) was obtained from Conservation Genetics CAS Kunming Cell Bank (Yunnan, China). These cells were cultivated in Dulbecco's modified eagle medium (DMEM; Invitrogen, Waltham, MA, USA) containing $10 \%$ fetal bovine serum (FBS; Invitrogen), $100 \mathrm{U} / \mathrm{mL}$ penicillin and $100 \mu \mathrm{g} / \mathrm{mL}$ streptomycin. The cells were grown in an incubator of $\mathrm{CO}_{2}(5 \%)$ at $37^{\circ} \mathrm{C}$.

For this study, small interfering RNA against LINC01410 (si-LINC01410) and the corresponding control (si-NC), miR-545-3p mimic (miR-545-3p) and the corresponding control (miR-NC), miR-545-3p inhibitor (anti-miR-545-3p) and the corresponding control (antimiR-NC), LINC01410 or HK2 overexpression plasmid (LINC01410 or HK2) and the corresponding control (pcDNA) were bought from GenePharma (Shanghai, China). Lentivirus-mediated shRNA interference targeting LINC01410 (sh-LINC01410) and the corresponding control (sh-NC) were constructed by Genechem (Shanghai, China). GI-LI-N and SK-N-BE(2) cells were transfected with oligonucleotide $(50 \mathrm{nM})$ or plasmid $(2 \mu \mathrm{g})$ using Lipofectamine 3000 (Invitrogen). 


\section{Quantitative Real-Time Polymerase Chain Reaction (qRT-PCR)}

TRIzol reagent (Invitrogen) was applied to isolate total RNA from tissues (NB tissues and normal tissues) and cells (HEK293, GI-LI-N and SK-N-BE(2)). Next, the first strand of complementary DNA (cDNA) was synthesized with a High-Capacity cDNA Reverse Transcription Kit and TaqMan MicroRNA Reverse Transcription Kit (Thermo Fisher Scientific, Waltham, MA, USA). The qRTPCR was carried out using the SYBR Green PCR kit (Thermo Fisher Scientific) on the ABI 7300 system (Thermo Fisher Scientific). In this study, the primers were as follows: LINC01410 (Forward, 5'-GTGACAAG AATGGCCCAAGC-3'; Reverse, 5'-ACTGTGCACCTG TTACACCA-3'); miR-545-3p (Forward, 5'-GCCGAG TCAGCAAACATTTAT-3'; Reverse, 5'-CAGTGCGT GTCGTGGAGT-3'); HK2 (Forward, 5'-ACAGCCT GGACGAGAGCATC-3'; Reverse, 5'-AGGTCAAACTCC TCTCGCCG-3'); glyceraldehyde-3-phosphate dehydrogenase (GAPDH) (Forward, 5'-CGCTCTCTGCTCCT CCTGTTC-3'; Reverse, 5'-ATCCGTTGACTC CGACCTTCAC-3'), U6 snRNA (Forward, 5'-CTCGC TTCGGCAGCACATATACT-3'; Reverse, 5'-ACGCTT CACGAATTTGCGTGTC-3'). The LINC01410, HK2 or miR-545-3p expression was evaluated with the $2^{-\Delta \Delta C t}$ method, followed by normalizing to GAPDH or U6 snRNA, respectively.

\section{Cell Viability Assay}

Methylthiazolyldiphenyl tetrazolium bromide (MTT) was utilized for detecting cell viability. In brief, GI-LI-N and SK-N-BE(2) cell suspension $(100 \mathrm{uL}, 2 \times 103$ cells) were seeded in 96-well plates and then transfected with siLINC01410, si-LINC01410 + anti-miR-545-3p, miR-545$3 p$, miR-545-3p + HK2, or matched controls. MTT reagent $(5 \mathrm{mg} / \mathrm{mL}, 10 \mu \mathrm{L}$, Beyotime, Shanghai, China) was added to each well after transfection. After incubation for $4 \mathrm{~h}$, the cultured medium from per well would be carefully discarded and dimethyl sulfoxide $(150 \mu \mathrm{L})$ was added. Lastly, the absorbance was evaluated at $490 \mathrm{~nm}$ under a microplate reader (Bio-Rad, Hercules, CA, USA).

\section{Irradiation (IR) and Colony Formation Assay}

After transfection for $48 \mathrm{~h}$, GI-LI-N and SK-N-BE(2) cells were placed into six-well plates and the medium was updated every three days. For treatment of IR, cells were exposed to different doses of radiation by a linear accelerator (Varian, Palo Alto, CA, USA) at a dose rate of $3.5 \mathrm{~Gy} / \mathrm{min}$. After incubation for 2 weeks, GI-LI-N and SK-N-BE(2) cells were carefully washed with cold phosphate-buffered saline (PBS; $\mathrm{pH}=7.2$ ) and subsequently fixed with paraformaldehyde (4\%) for $30 \mathrm{~min}$ at $4^{\circ} \mathrm{C}$. After that, GI-LI-N and SK-N-BE (2) cells were washed by PBS and then stained by crystal violet $(0.1 \%$, Sigma-Aldrich, St. Louis, MO, USA). Microscope (Olympus, Tokyo, Japan) was applied to count colonies containing more than 50 cells. The survival fraction was calculated following the equation: the number of colonies/(number of plated cells $\times$ plating efficiency). ${ }^{24}$

\section{Transwell Assay}

Transwell assay was performed using a 24-well plate inserts with $8 \mu \mathrm{m}$ pores (Corning Incorporated, Corning, NY, USA) to evaluate cell invasion capacity. GI-LI-N and SK-N-BE(2) cells $\left(2 \times 10^{4}\right.$ cells/well $)$ re-suspended in DMEM medium alone $(100 \mu \mathrm{L})$ were placed into the top chamber pre-coated with Matrigel (BD Bioscience, Franklin Lakes, NJ, USA). To induce cells invading through the membrane, the bottom chamber was filled with DMEM containing 10\% FBS. After $24 \mathrm{~h}$ of incubation, non-invaded cells were carefully removed with cotton bud, and invaded cells were fixed using ethanol (95\%) and then stained using crystal violet $(0.1 \%)$. Finally, a microscope (Olympus, Tokyo, Japan) was applied to observe and count invaded cells in five random fields.

\section{Measurement of Glucose Consumption and Lactate Production}

In accordance with the manufacturer's instructions, the glucose consumption and lactate production were detected with the glucose assay kit (Sigma-Aldrich) and lactate assay kit (BioVision, Mountain View, CA, USA), respectively. A standard calibration curve was used for determining the data, followed by normalizing to the amount of total protein. The consumption glucose and lactate production were analyzed by detecting the absorbance of each tube against the reagent blank at $540 \mathrm{~nm}$ and $570 \mathrm{~nm}$, respectively.

\section{Dual-Luciferase Reporter Assay}

The possible binding sites of miR-545-3p and LINC01410 or HK2 were predicted by starBase v2.0. The LINC01410 and HK2 3'UTR fragments that contained wild-type (WT) or mutant (MUT) binding sites of miR-545-3p were amplified and inserted into pmirGlO luciferase reporter vector 
(Promega, Madison, WI, USA) to create the WT plasmids (WT-LINC01410, HK2 3'UTR-WT) or MUT plasmids (MUT-LINC01410, HK2 3'UTR-MUT). Afterward, GI-LI -N and SK-N-BE(2) cells were co-transfected with reporter plasmids and miR-545-3p or miR-NC. After incubating the cells for $48 \mathrm{~h}$, the luciferase activity was estimated via DualLuciferase Reporter assay system (Promega).

\section{RNA Immunoprecipitation (RIP) Assay}

EZ-Magna RIP Kit (Millipore, Billerica, MA, USA) was used to validate the interaction between miR-545-3p and LINC01410 or HK2. In brief, cells were lysed by complete RIP lysis buffer. After that, whole cell extract $(100 \mu \mathrm{L})$ was incubated by RIP buffer containing magnetic beads coated with human anti-argonaute2 (Ago2) or antiimmunoglobulin G (IgG) (as control). Next, samples were digested using the proteinase K. Finally, the RNAs in the immunoprecipitates were isolated with Trizol reagent, and the expression levels of LINC01410, miR545-3p and HK2 were examined by qRT-PCR.

\section{RNA Pull-Down Assay}

RNA pull-down assay was performed to verify the endogenous interaction between miR-545-3p and LINC01410 or HK2 in NB cells. Biotinylated miRNAs (bio-miR-NC and bio-miR-545-3p) were obtained from Sangon Biotech (Shanghai, China). In brief, cells transfected with bio-miRNC or bio- miR-545-3p were lysed by lysis buffer. The cell lysates were incubated with Dynabeads M-280 Streptavidin (Thermo Fisher Scientific). Next, the beads were washed, the RNA complexes bound to the beads were extracted with Trizol reagent. Finally, the enrichment of LINC01410 and HK2 was detected by qRT-PCR.

\section{Western Blot Assay}

Transfected cells were lysed using RIPA lysis buffer (SigmaAldrich) supplemented with $1 \mathrm{mM}$ phenylmethylsulfonyl fluoride (PMSF; Sigma-Aldrich) to extract the total protein. After quantification by using bicinchoninic acid protein assay kit (Tanon, Shanghai, China), $30 \mu \mathrm{g}$ of protein in each group was separated using sodium dodecyl sulfate-polyacrylamide gel electrophoresis (SDS-PAGE). After that, polyvinylidene fluoride (Beyotime) membranes were employed to transfer the protein. After blocking with 5\% non-fat milk in PBS containing $0.05 \%$ Tween 20 (PBST), the membranes were immunoblotted by primary antibody against HK2 (1:5000, ab227198, Abcam, Cambridge, UK) or GAPDH (1:2500, ab9485, Abcam) for $12-16 \mathrm{~h}$ at $4^{\circ} \mathrm{C}$. Afterward, membranes were maintained in horseradish peroxidase-conjugated antirabbit IgG (1:4000, D110058, Sangon Biotech) after washing with PBST. Immunoreactive bands were visualized through the enhanced chemiluminescence (ECL; Tanon) reagent following the manufacturer's protocol. ImageJ software was utilized to quantify the protein expression. GAPDH was used as an internal control.

\section{In vivo Tumor Growth Assay}

$\mathrm{BALB} / \mathrm{c}$ nude mice (male, 5-week-old) were purchased from Vital River Laboratory Animal Technology Co., Ltd, (Beijing, China). The sh-LINC01410 or sh-NC was transfected into SK-N-BE(2) cells. Subsequently, stably transfected cells $\left(3 \times 10^{6}\right)$ were subcutaneously injected in $\mathrm{BALB} / \mathrm{c}$ nude mice. From the 8th day, tumor volume was examined using a caliper every 4 days and calculated with the formula: length $\times$ width $^{2} / 2$. After injection for 10 days, sh-LINC01410 group or sh-NC group were randomly divided into two groups. One group was irradiated with 6Gy X-ray once a week, and the other group served as control. All mice were sacrificed after injection for 4 weeks, and tumor samples were weighted and collected for detection of LINC01410, miR-545-3p and HK2 expression levels. The animal experiments were approved by the Committee on the Ethics of Animal Experiments of People's Hospital of Rizhao. All animal procedures were conducted following the Guidelines for Care and Use of Laboratory Animals of "National Institutes of Health".

\section{Immunohistochemistry (IHC) Analysis}

Tumor tissues from transplanted nude mice were fixed in paraformaldehyde (4\%, Beyotime) and then were dehydrated, embedded in paraffin, and cut. After that, consecutive tumor sections ( $4 \mu \mathrm{m}$ in thickness) were incubated by antibodies against $\mathrm{HK} 2$ (1:200, ab227198, Abcam) overnight at $4^{\circ} \mathrm{C}$, followed by incubation with secondary antibody (1:5000, ab205718, Abcam) for $1 \mathrm{~h}$. The sections were stained by diaminobenzidine (DAB), and cell nucleus was counterstained with haematoxylin. At last, stained sections were photographed using a fluorescence microscope (Olympus).

\section{Statistical Analysis}

All data in this study were displayed as mean \pm standard deviation (SD) and all experiments were repeated at least 3 times. Correlation between miR-545-3p and LINC01410 or HK2 was detected by Spearman rank correlation. The results from different groups were assessed using the two-tailed Student's $t$-test (for two groups) and a one-way analysis of 
variance (ANOVA; for more than two groups). Statistical analyses were performed using GraphPad Prism version 6.0 software (GraphPad Software, San Diego California, USA). $P<0.05$ indicates a statistically significant result. $\left({ }^{*} P<0.05\right.$, $* * P<0.01, * * * P<0.001, * * * * P<0.0001)$.

\section{Results}

\section{LINCOI4I 0 Was Upregulated and miR-545-3p Was Downregulated in NB Tissues and Cells}

Firstly, we explored the expression of LINC01410 and miR-545-3p in NB tissues and cells by qRT-PCR. As presented in Figure 1A and B, LINC01410 was overexpressed in NB tissues and cells in contrast to their matched controls. Moreover, we found that the expression of miR545-3p was decreased in NB tissues and cells compared to their corresponding controls (Figure 1C and D). Besides, the correlation between of LINC01410 and miR-545-3p was analyzed in NB tissues. As depicted in Figure 1E, LINC01410 expression was negatively correlated with miR-545-3p in NB tissues $(r=-0.5852, p<0.001)$. These data indicated that LINC01410 and miR-545-3p might be involved in the pathogenesis of NB.

\section{LINCOI4I0 Knockdown Inhibited Cell} Proliferation and Invasion and Enhanced Radiosensitivity by Inhibiting Glycolysis in NB Cells

Next, the effects of LINC01410 on cell proliferation, invasion, glycolysis, and radiosensitivity were investigated using lossfunction experiments. The transfection efficiency of siLINC01410 was determined by qRT-PCR. The results showed that the expression of LINC01410 was decreased in GI-LI-N and SK-N-BE(2) cells transfected with si-LINC01410 compared to those cells transfected with si-NC or control cells (Figure 2A), suggesting the transfection of si-LINC01410 was successful. MTT assay revealed that LINC01410 interference decreased GI-LI-N and SK-N-BE(2) cell viability (Figure 2B). Moreover, colony formation assay indicated that LINC01410 deficiency decreased the number of colonies in GI-LI-N and SK-N-BE(2) cells (Figure 2C). Furthermore, silencing LINC01410 repressed GI-LI-N and SK-N-BE(2) cell invasion ability (Figure 2D). GI-LI-N and SK-N-BE(2) cells were transfected with si-LINC01410 or si-NC and then irradiated with 0 Gy to 8 Gy to explore the effect of LINC01410 on radiosensitivity. The data showed that LINC01410 downregulation strikingly decreased the survival fraction of GI-LI-N

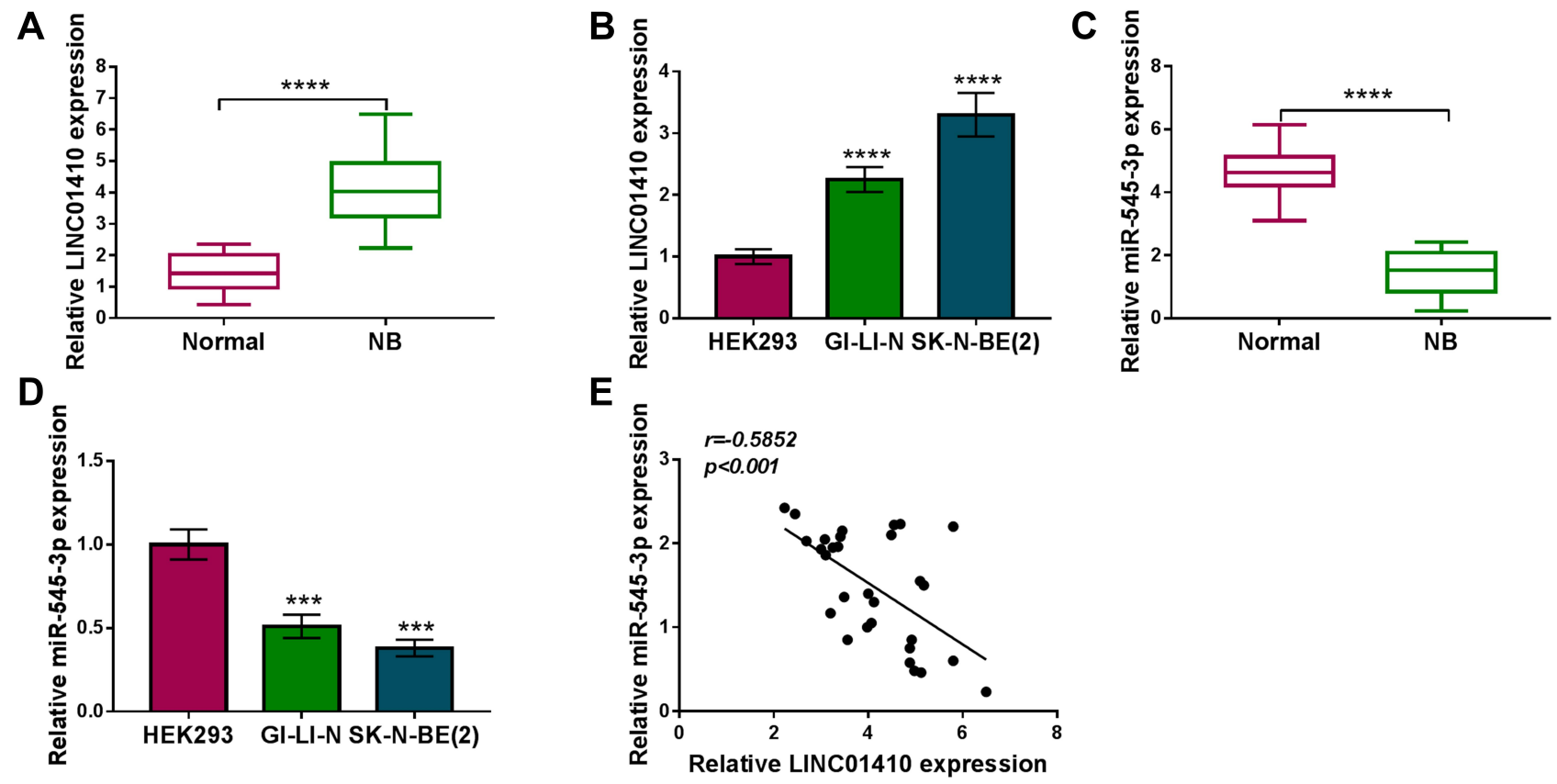

Figure I LINC0I4I0 was upregulated while miR-545-3p was downregulated in NB tissues and cell lines. (A and B) Relative LINC0I4I0 expression was detected by qRTPCR in NB tissues, normal tissues, NB cells (GI-LI-N and SK-N-BE(2)) and HEK293 cells. (C and D) Relative miR-545-3p expression was measured by qRT-PCR in NB tissues, normal tissues, NB cells (GI-LI-N and SK-N-BE(2)) and HEK293 cells. (E) Correlation between LINC0I4I0 and miR-545-3p expression was verified by Spearman rank correlation. $* * * P<0.001$, $* * * * P<0.0001$. 

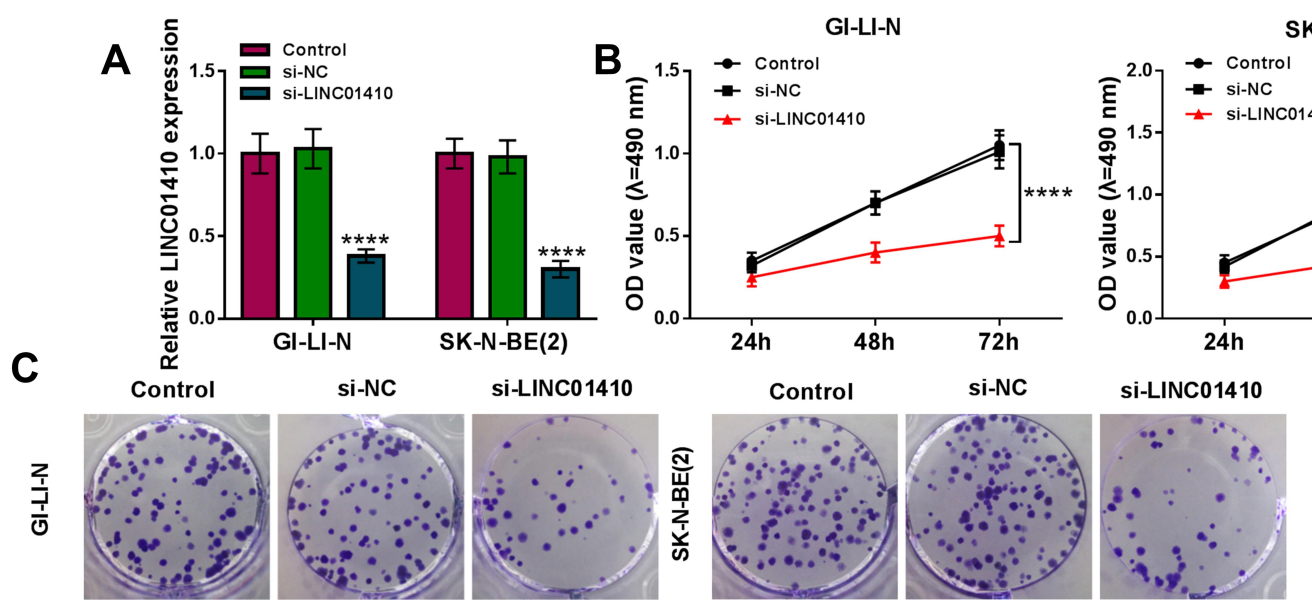

SK-N-BE(2)
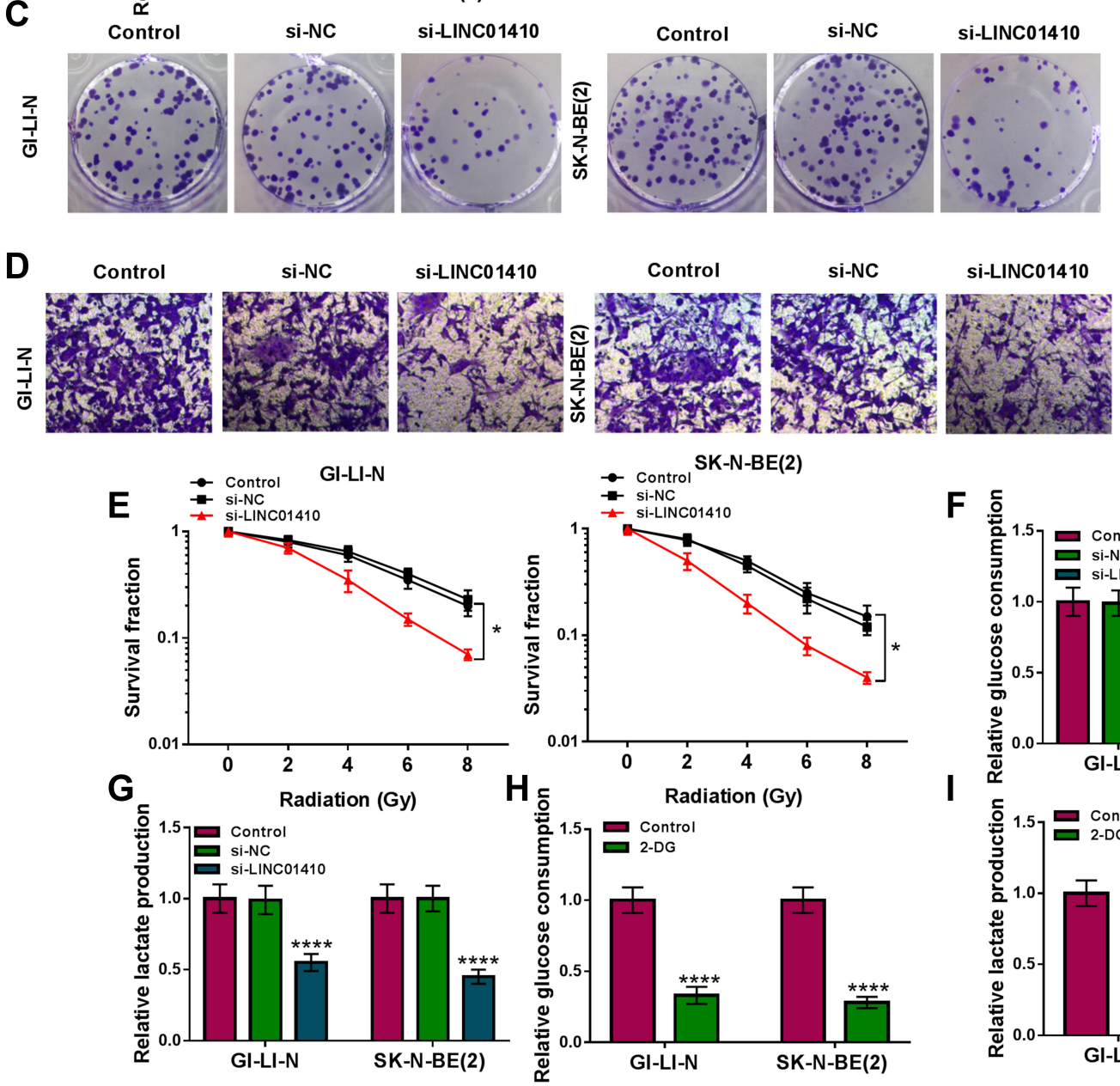

$\mathbf{F}$
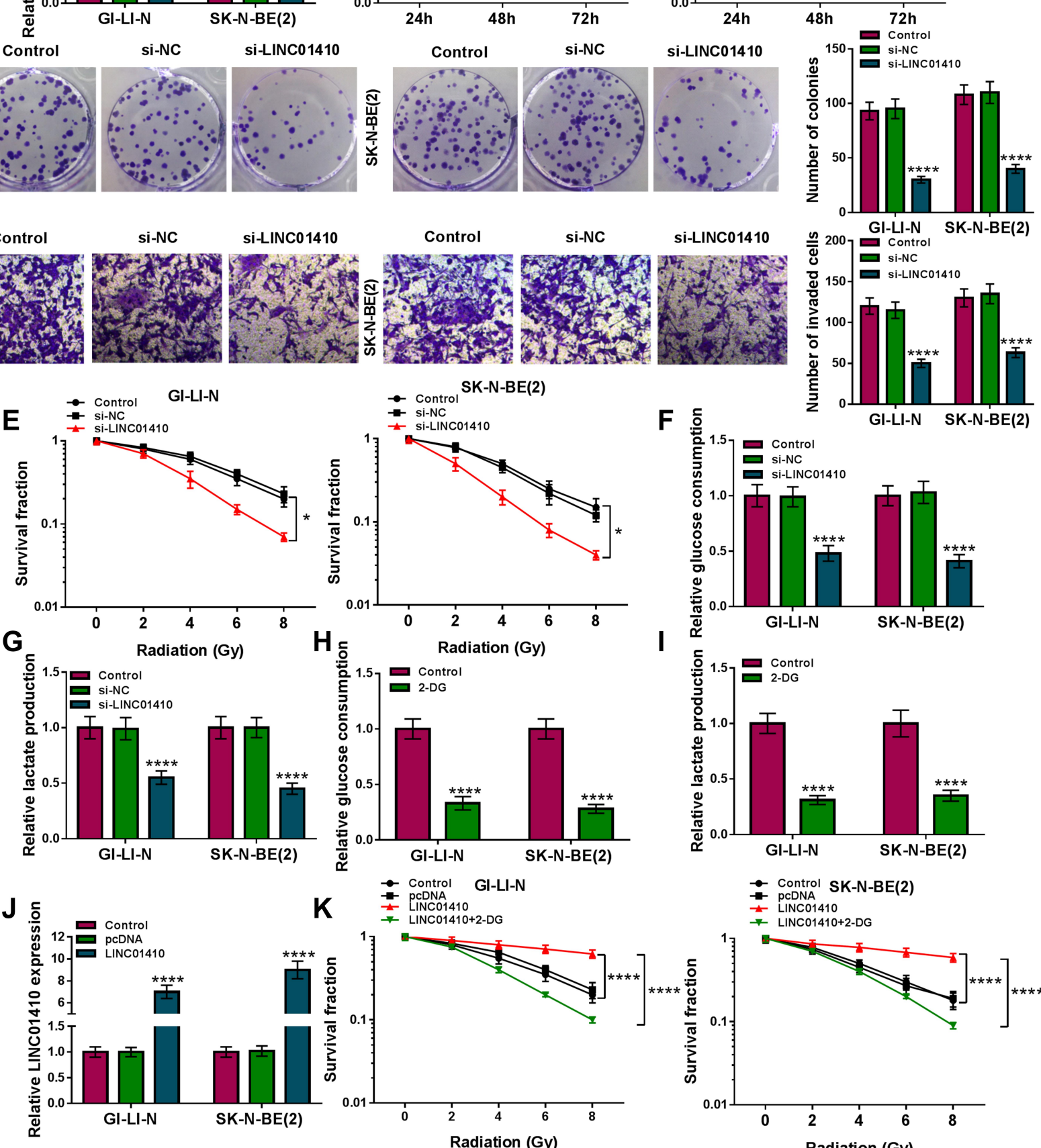

I
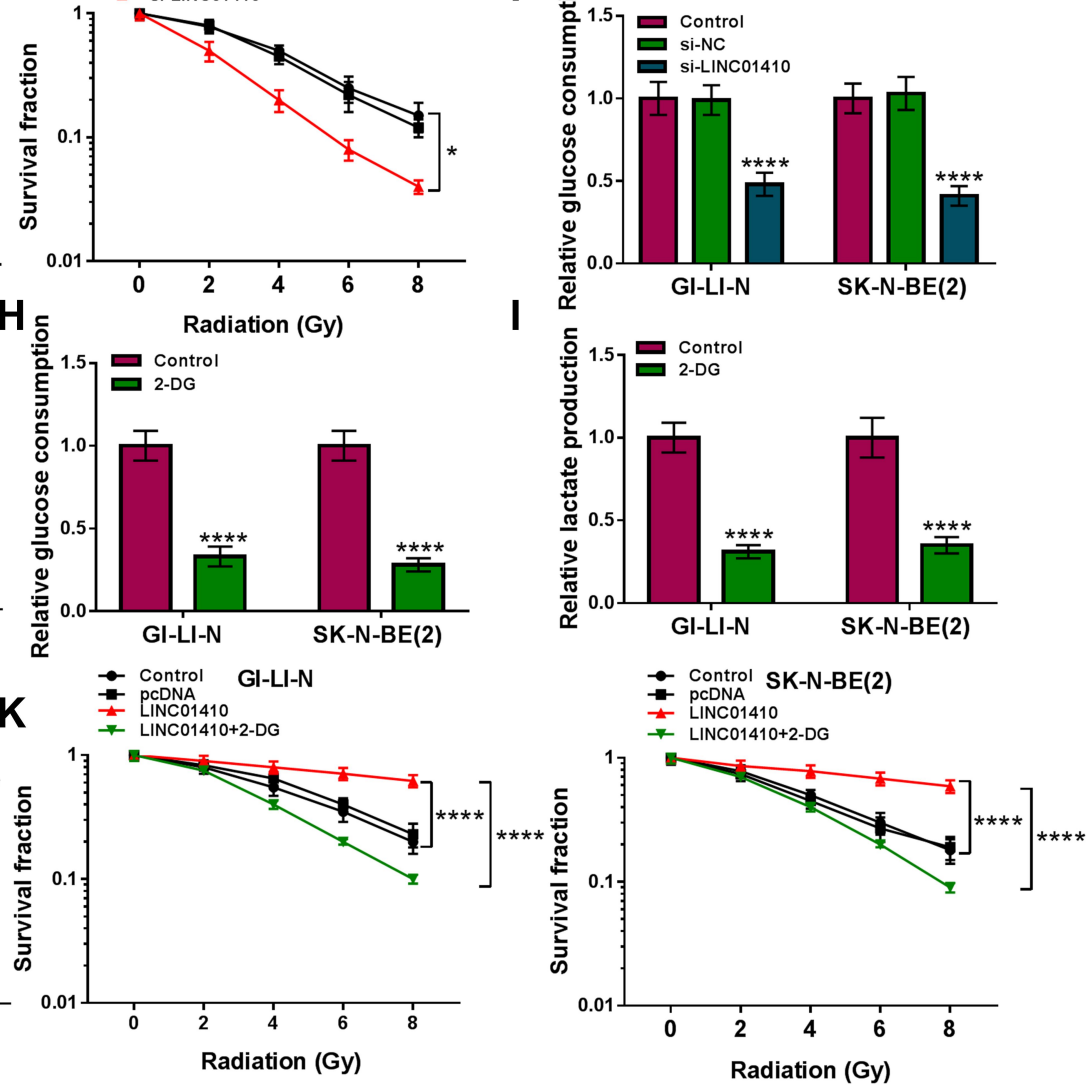

Figure 2 LINC0I4I0 knockdown suppressed cell proliferation and invasion, and promoted radiosensitivity through inhibiting glycolysis in NB cells. (A-D) GI-LI-N and SKN-BE(2) cells were divided into 3 groups: Control, si-NC, and si-LINC0I4IO. (A) The expression of LINC0I4I0 was examined using qRT-PCR analysis. (B) MTT assay was employed to analyze cell viability. (C) Colony formation assay was utilized to determine the number of colonies (incubation for 14 days). (D) Cell invasion was detected by transwell assay (incubation for $24 \mathrm{~h}$ ). (E) Cell survival fraction was detected by colony formation assay in GI-LI-N and SK-N-BE(2) cells transfected with si-NC or siLINCOI4I0 and exposed to radiation (incubation for I4 days). (F-I) Glucose consumption and lactate production were measured in GI-LI-N and SK-N-BE(2) cells transfected with si-NC or si-LINCOI4IO as well as the cells treated with 2-DG by glucose assay kit and lactate assay kit, respectively. (J) The expression of LINCOI4I0 was analyzed in GI-LI-N and SK-N-BE(2) cells transfected with pcDNA or LINCOI4I0 using qRT-PCR analysis. (K) Colony formation assay was applied to determine cell survival fraction in LI-N and SK-N-BE(2) cells transfected with si-NC or si-LINC0I4I0 or si-LINC0I4I0 + 2-DG under radiation condition. $* P<0.05$, $* * * * P<0.0001$. 
and SK-N-BE(2) cells exposed to radiation compared to si-NC or control group (Figure 2E). Most cancer cells mainly rely on aerobic glycolysis to produce the energy needed for cellular processes. ${ }^{25}$ This aerobic glycolysis will result in increased glucose consumption and lactate production. We observed that knockdown of LINC01410 inhibited glucose consumption and lactate production in GI-LI-N and SK-N-BE(2) cells (Figure $2 \mathrm{~F}$ and $\mathrm{G}$ ). Moreover, we found that glucose consumption and lactate production were decreased in GI-LI-N and SK-N-BE(2) cells treated with 2-deoxyglucose (2-DG; glycolytic inhibitor) (Figure 2H and I). QRT-PCR analysis showed that the expression of LINC01410 was enhanced in cells transfected with LINC01410 compared to pcDNA group (Figure 2J). Besides, the promotive effect of LINC01410 on cell survival fraction was overturned by treatment with 2-DG (Figure 2K), suggesting that LINC01410 knockdown enhanced the radiosensitivity through inhibiting glycolysis. Taken together, these results demonstrated that LINC01410 interference inhibited the tumorigenesis and increased the radiosensitivity in NB cells.

\section{LINCO I 4 I0 Directly Interacted with miR-545-3p in NB Cells}

In view of the alteration of LINC01410 and miR-545-3p in NB tissues and cell lines and the negative correction between LINC01410 and miR-545-3p expression, we wondered whether the function of LINC01410 was mediated by miR545-3p through complementary binding sites. StarBase v2.0 predicted that LINC01410 contained putative binding sites of miR-545-3p (Figure 3A). Transfection of miR-545-3p increased the expression of miR-545-3p expression, while transfection of anti-miR-545-3p decreased miR-545-3p expression in GI-LI-N and SK-N-BE(2) cells (Figure 3B). To verify whether miR-545-3p was a direct target of LINC01410, the dual-luciferase reporter assay, RIP assay and RNA pull-down assay were performed. As presented in Figure 3C, overexpression of miR-545-3p led to a significant decrease in the luciferase activity of WT-LINC01410, while luciferase activity of MUT-LINC01410 was not evidently affected after transfection with miR-545-3p in GI-LI-N and SK-N-BE(2) cells. RIP assay revealed that both miR-545-3p and LINC01410 were immunoprecipitated in argonaute2 (Ago2) group instead of immunoglobulin $\mathrm{G}(\mathrm{IgG})$ group (Figure 3D). RNA pull-down assay showed that the expression of LINC01410 was significantly elevated in biotinylated miR-545-3p (bio-miR-545-3p) group rather than biotinylated negative (bio-miR-NC) group (Figure 3E), suggesting that
miR-545-3p could pull down LINC01410. Moreover, the expression of LINC01410 was reduced in GI-LI-N and SK$\mathrm{N}-\mathrm{BE}(2)$ cells transfected with si-LINC01410, while its expression was elevated in cells transfected with LINC01410 (Figure 3F). Next, we explored the effect of LINC01410 on expression of miR-545-3p. As expected, knockdown of LINC01410 increased the expression of miR-545-3p, whereas overexpression of LINC01410 presented an opposite effect (Figure 3G), suggesting that miR-545-3p was negatively regulated by LINC01410. Meanwhile, we observed that cotransfection of anti-miR-545-3p and si-LINC01410 abated the effect of si-LINC01410 on promotion of miR-545-3p expression (Figure 3H). Additionally, inhibition of miR-545$3 p$ reversed the inhibitory effects of LINC01410 knockdown on viability, colony formation and invasion of GI-LI-N and SK-N-BE(2) cells (Figure 3I-K). Moreover, the suppressive effect of LINC01410 interference on the survival fraction was abolished by downregulation of miR-545-3p in GI-LI-N and SK-N-BE(2) cells exposed to IR (Figure 3L). Furthermore, miR-545-3p silence abated the inhibitory effect of LINC01410 knockdown on glucose consumption and lactate production (Figure 3M and N). Our findings suggested that miR-545-3p could bind to LINC01410 and its knockdown reversed the effects of LINC01410 interference on tumorigenesis and radiosensitivity in NB cells.

\section{HK2 Was a Direct Target of miR-545-3p in NB Cells}

To explore the underlying mechanism of miR-545-3p in progression of $\mathrm{NB}$, the function targets of miR-545-3p were searched by starBase v2.0. As presented in Figure 4A, miR545-3p and HK2 3'UTR had complementary binding sequence, indicating that HK2 might be a target of miR-545$3 p$. Dual-luciferase reporter assay displayed that the luciferase activity of HK2 3'UTR-WT was obviously inhibited by transfection of miR-545-3p, whereas no clear change of the luciferase activity of HK2 3'UTR-MUT was found (Figure 4B). RIP assay demonstrated that miR-545-3p and HK2 were obviously enriched in the Ago2 immunoprecipitation compared with the IgG immunoprecipitation (Figure 4C). Moreover, HK2 was strikingly enriched in GI-LI-N and SK-N-BE(2) cells transfected with bio-miR-545-3p (Figure 4D). Next, we detected the expression of HK2 in NB tissues and cells. The results suggested that the mRNA expression and protein expression of HK2 were increased in NB tissues in comparison with normal tissues (Figure 4E and F). Additionally, HK2 expression was negatively correlated with miR-545-3p level in NB 


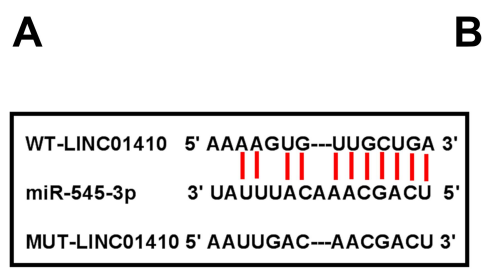

D

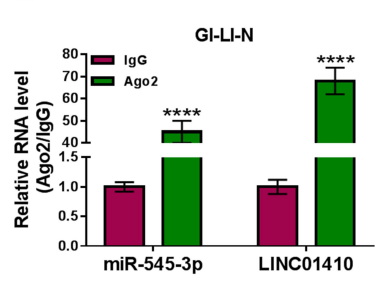

F
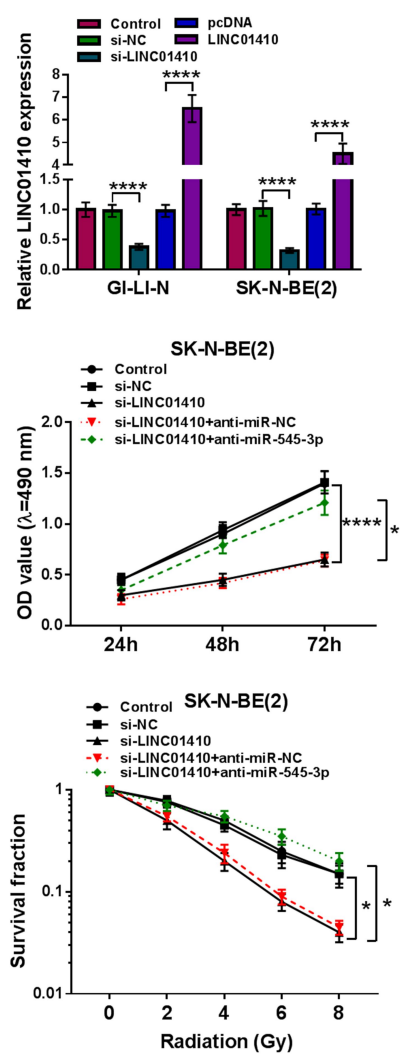
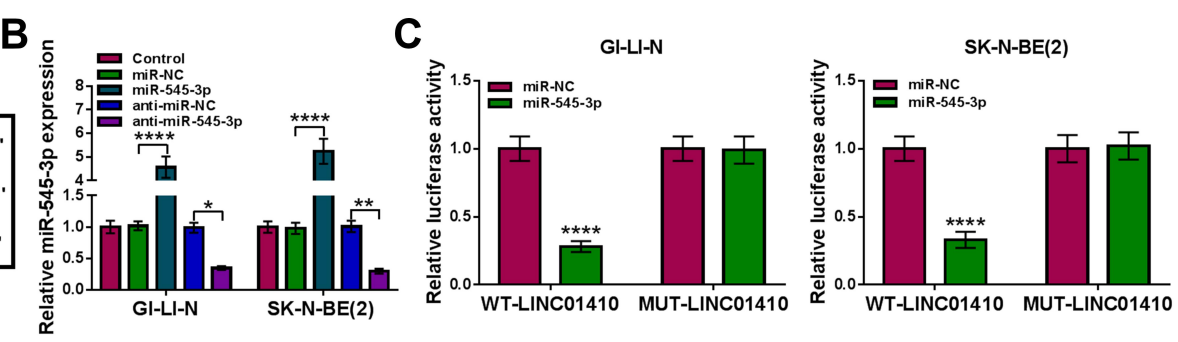

E

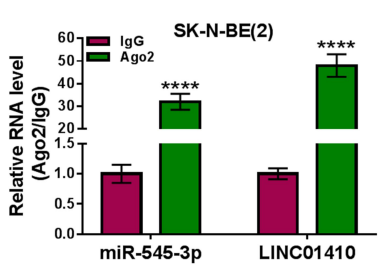

G

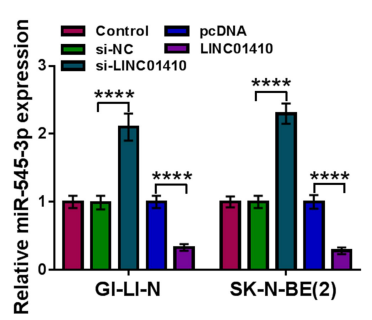

J

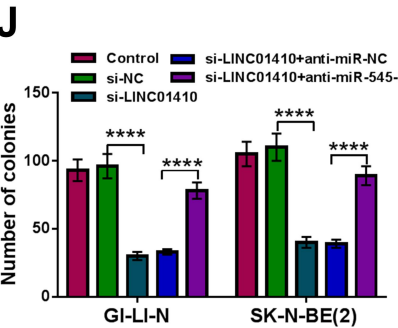

M

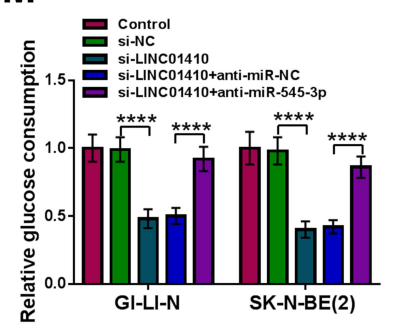

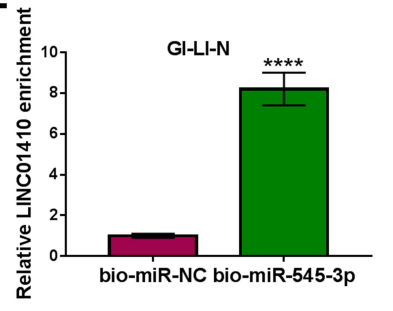

H

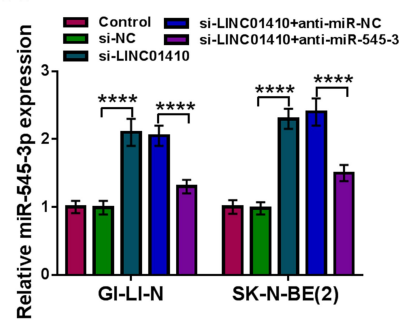

$\mathrm{K}$

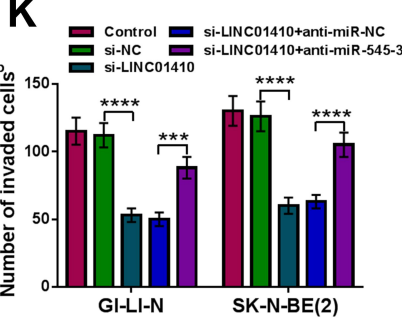

N

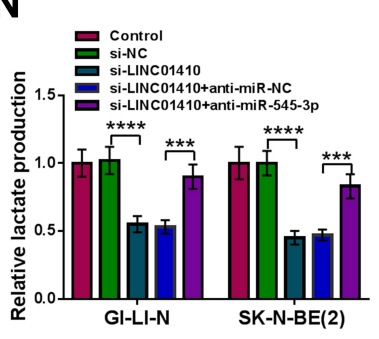

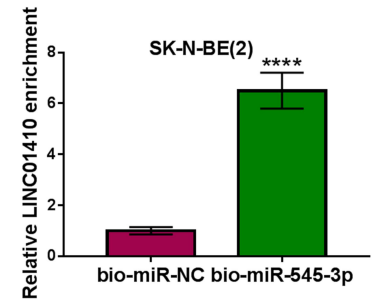

|

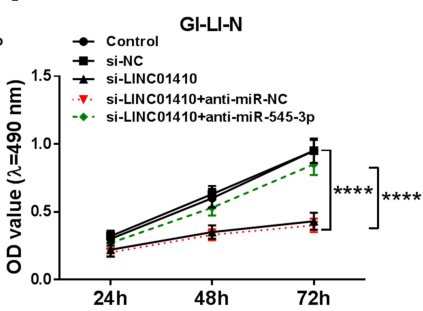

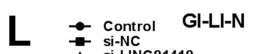

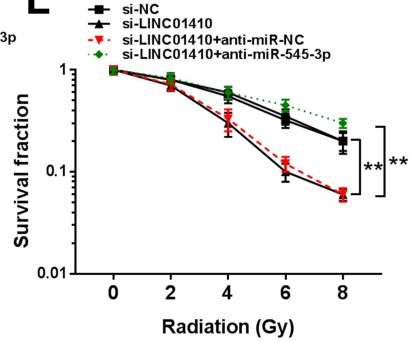

Figure 3 LINC01410 knockdown inhibited tumorigenesis and promoted radiosensitivity in NB cells by sponging miR-545-3p. (A) Predicted binding sites between miR-545$3 p$ and LINCOI4I0 were shown. (B) The expression of miR-545-3p was examined by qRT-PCR in GI-LI-N and SK-N-BE(2) cells transfected with miR-NC, miR-545-3p, antimiR-NC, or anti-miR-545-3p. (C) Dual-luciferase reporter assay was conducted to determine luciferase activity in Gl-LI-N and SK-N-BE(2) cells co-transfected with miR545-3p or miR-NC and WT-LINC0I4IO or MUT-LINC0I4I0. (D) The levels of miR-545-3p and LINC0I4I0 were measured by RIP assay in GI-LI-N and SK-N-BE(2) incubated with Ago2 or IgG. (E) The level of LINCOI4I 0 was examined in GI-LI-N and SK-N-BE(2) cells transfected with bio-miR-545-3p or bio-miR-NC by RNA pull-down assay. (F and $\mathbf{G}$ ) LINCOI4I0 and miR-545-3p expression were detected using qRT-PCR analysis in GI-LI-N and SK-N-BE(2) cells transfected with si-NC, si-LINC0I4I0, PCDNA, or LINCOI4I0. (H-L) GI-LI-N and SK-N-BE(2) cells were transfected with si-NC, si-LINC0I4I0, si-LINCOI4I0 + anti-miR-NC, or si-LINCOI4IO + anti-miR-5453p. (H) The expression of miR-545-3p was measured by qRT-PCR. (I) MTT assay was performed to examine cell viability. (J) The number of colonies was determined by colony formation assay (incubation for 14 days). (K) Transwell assay was carried out to detect the number of invaded cells (incubation for $24 \mathrm{~h}$ ). (L) Cell survival fraction was determined by colony formation assay under radiation condition (incubation for 14 days). ( $\mathbf{M}$ and $\mathbf{N}$ ) Glucose consumption or lactate production was measured by glucose assay kit or lactate assay kit, respectively. $* P<0.05$, $* * P<0.01$, $* * * P<0.001$, $* * * * P<0.00001$. 

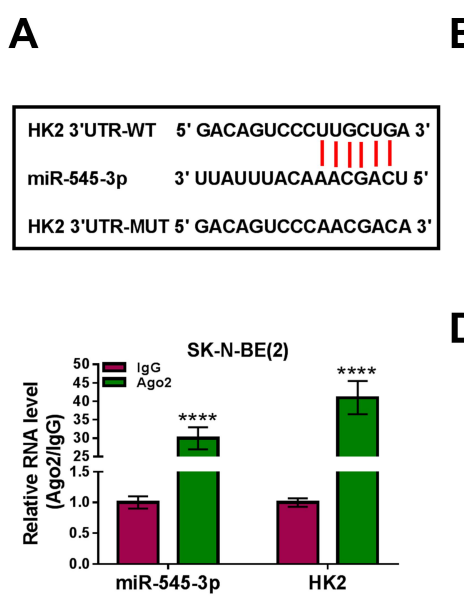

\section{$\mathbf{F}$}

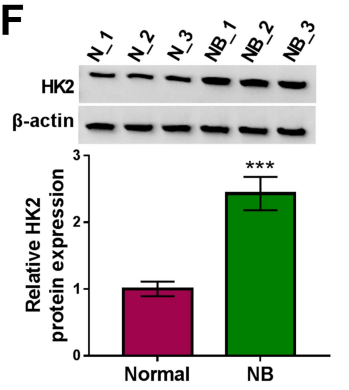

B

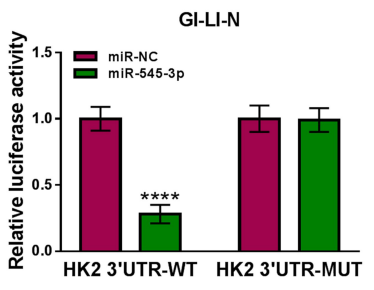

D

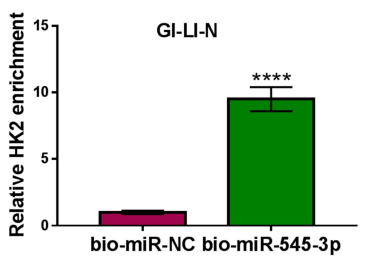

G

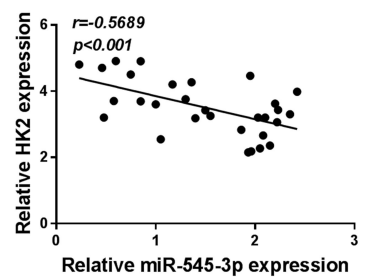

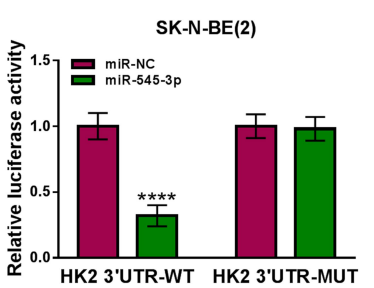

C

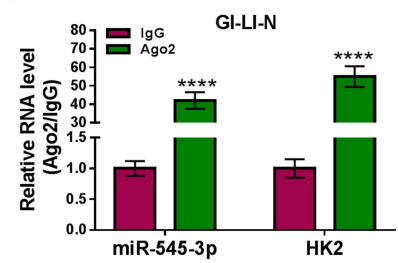

E

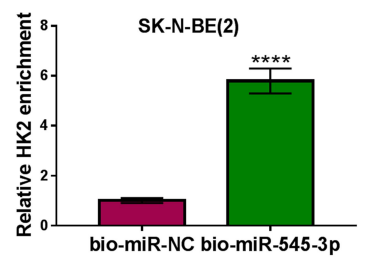

H
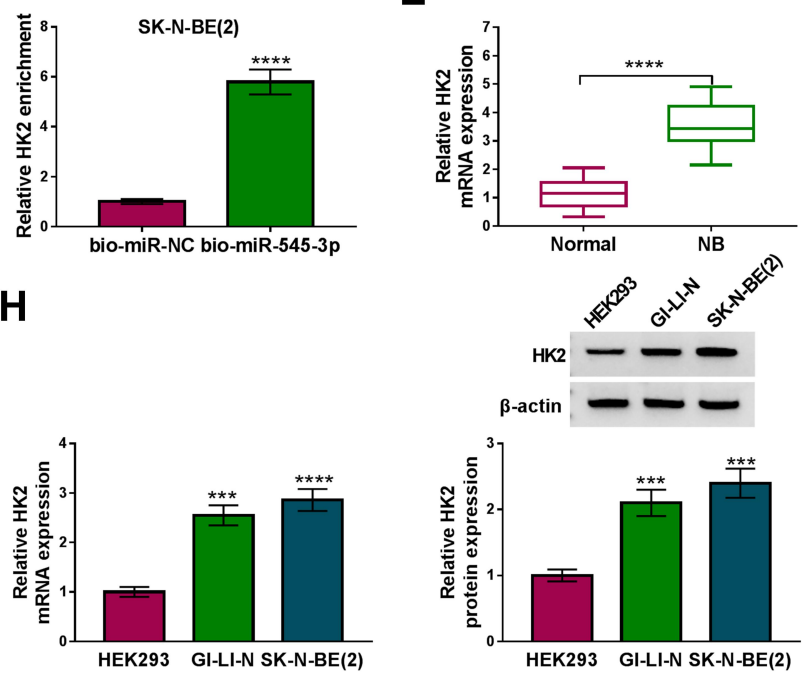

Figure 4 HK2 was a downstream target of miR-545-3p in NB cells. (A) The putative binding sites between miR-545-3p and HK2 were predicted by starBase v2.0. (B) Relative luciferase activity was determined in GI-LI-N and SK-N-BE(2) cells co-transfected with HK2 3'UTR-WT or HK2 3'UTR-MUT and miR-545-3p or miR-NC. (C) RIP assay was used to explore the interaction between HK2 and miR-545-3p in GI-LI-N and SK-N-BE(2) cells. (D) RNA pull-down assay was performed to confirm the targeted relationship between miR-545-3p and HK2 in GI-LI-N and SK-N-BE(2) cells. (E and F) The mRNA and protein expression of HK2 were evaluated in NB tissues and normal tissues by miR-545-3p and Western blot analysis, respectively. $(\mathbf{G})$ Correlation between HK2 and miR-545-3p expression was determined by Spearman rank correlation. $(\mathbf{H})$ The mRNA and protein expression of HK2 were assessed in NB cells (GI-LI-N and SK-N-BE(2)) and HEK293 cells by qRT-PCR and Western blot, respectively. ***P<0.00I, $* * * * p<0.000$ I.

tissues ( $\mathrm{r}=-0.5689, \mathrm{p}<0.001$ ) (Figure 4G). Likewise, the mRNA expression and protein expression of HK2 were also enhanced in NB cells (GI-LI-N and SK-N-BE(2)) compared to HEK293 cells (Figure 4H). These data illustrated that miR545-3p directly interacted with HK2.

\section{Overexpression of HK2 Reversed the Effects of miR-545-3p on Inhibition of Tumorigenesis and Promotion of Radiosensitivity in NB Cells}

To determine whether the effects of miR-545-3p were regulated by HK2 expression, GI-LI-N and SK-N-BE(2) cells were transfected with miR-NC, miR-545-3p, miR-545-3p + pcDNA, or miR-545-3p + HK2. Western blot showed that HK2 protein expression was decreased in cells transfected with miR-545-3p, while the effect was abated by co-transfection of HK2 (Figure 5A). Additionally, overexpression of miR-545-3p reduced the cell viability, colony formation and invasion of GI-LI-N and SK$\mathrm{N}-\mathrm{BE}(2)$ cells, which was reversed by upregulating HK2 (Figure
5B-D). Moreover, the inhibitory effects of miR-545-3p upregulation on survival fraction and glycolysis were also overturned by overexpression of HK2 (Figure 5E-H). Collectively, these findings demonstrated that miR-545-3p exerted its biological functions through regulating HK2 expression.

\section{HK2 Was Regulated by LINCOI4IO and miR-545-3p in NB Cells}

Next, knockdown efficiency of miR-545-3p was tested by qRTPCR. As shown in Figure 6A, the expression of miR-545-3p was significantly reduced in GI-LI-N and SK-N-BE(2) cells transfected with anti-miR-545-3p compared to those cells transfected with anti-miR-NC or control group. To explore whether LINC01410 served as a ceRNA of miR-545-3p to modulate HK2 expression, GI-LI-N and SK-N-BE(2) cells were transfected with si-NC, si-LINC01410, si-LINC01410 + anti-miR$\mathrm{NC}$, or si-LINC01410 + anti-miR-545-3p. Western blot assay demonstrated that the protein level of $\mathrm{HK} 2$ was decreased after transfection with si-LINC01410, while the effect was reversed by 

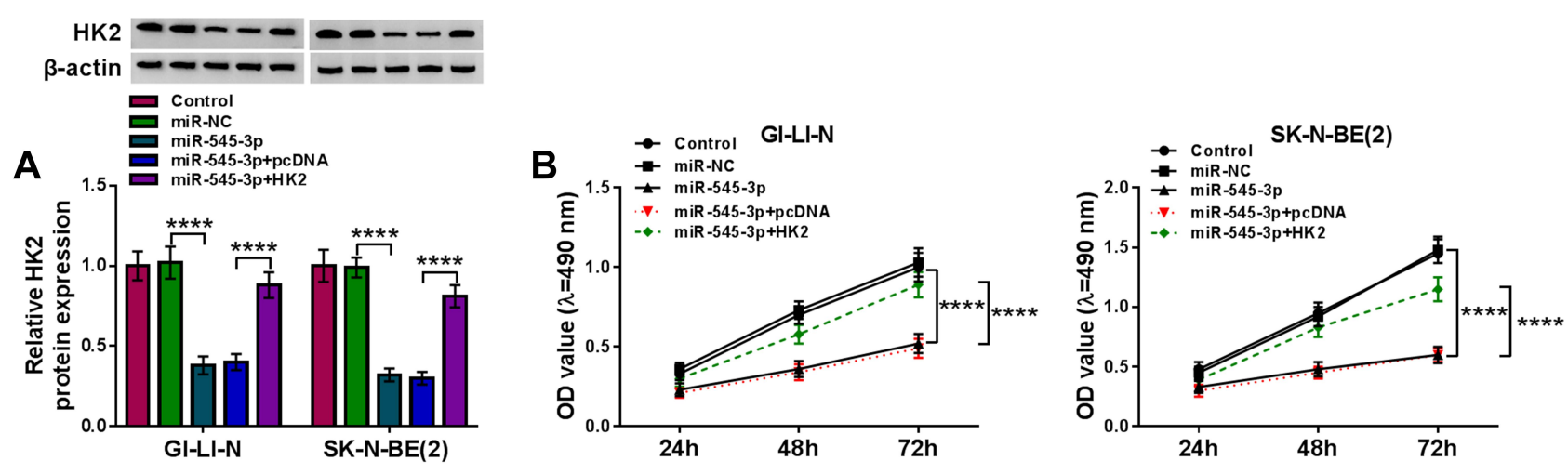

C

D

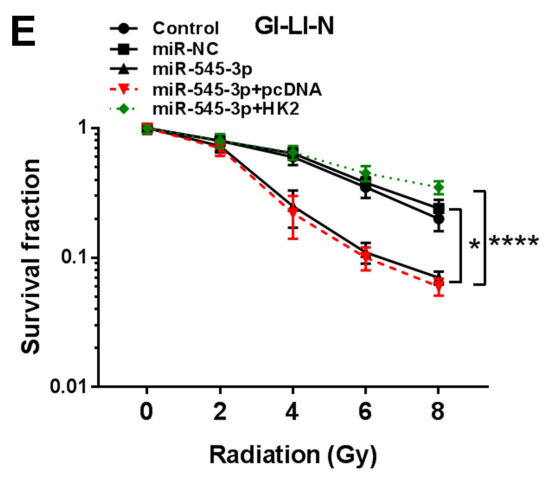

$\mathbf{F}$
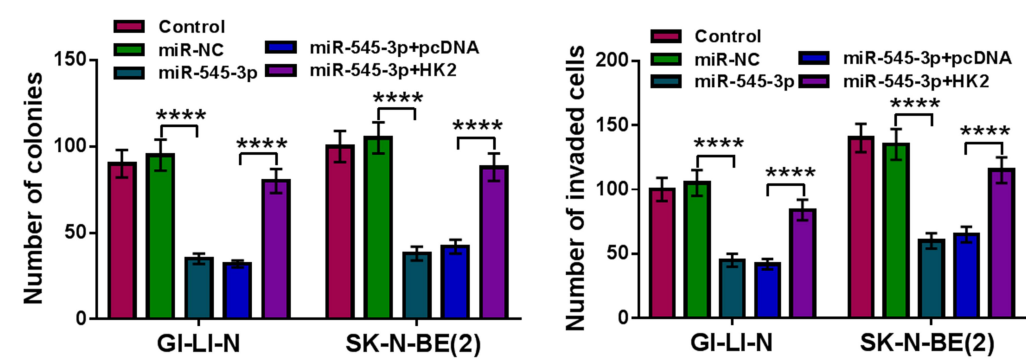

H

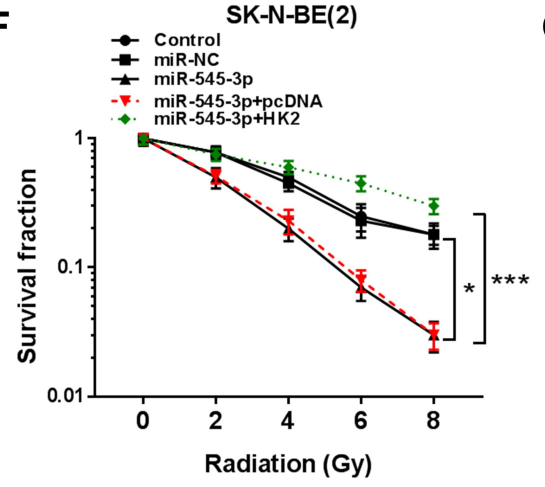

G
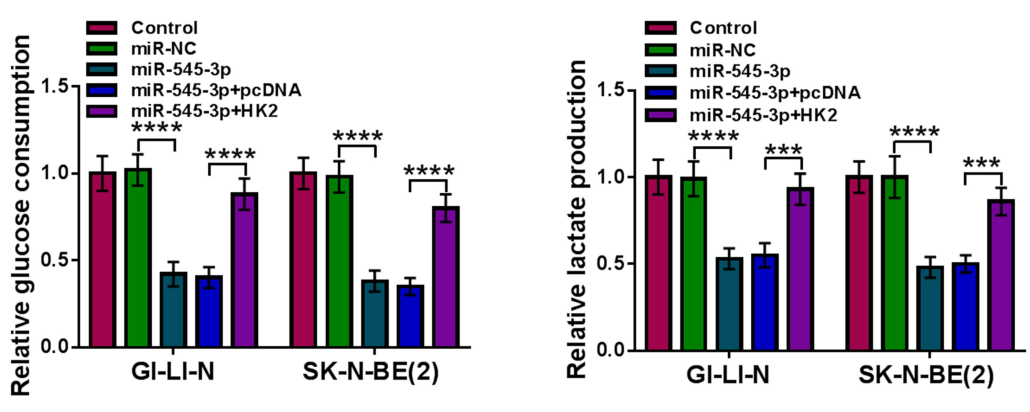

Figure 5 Overexpression of miR-545-3p suppressed tumorigenesis and increased radiosensitivity in NB cells by downregulating HK2. GI-LI-N and SK-N-BE(2) cells were divided into 5 groups: Control, miR-NC, miR-545-3p, miR-545-3p + pcDNA, and miR-545-3p + HK2. (A) The protein abundance of HK2 was detected by Western blot assay. (B) Cell viability was evaluated by MTT assay. (C) The number of colonies was determined using colony formation assay (incubation for I4 days). (D) Transwell assay was performed to assess cell invasion ability (incubation for $24 \mathrm{~h}$ ). (E and $\mathbf{F}$ ) Cell survival fraction was measured by colony formation assay under radiation condition (incubation for 14 days). ( $\mathbf{G}$ and $\mathbf{H}$ ) Glucose consumption and lactate production were measured using glucose assay kit and lactate assay kit, respectively. $* P<0.05$, $* * * P<0.001, * * * * P<0.0001$.

downregulating miR-545-3p (Figure 6B). In a word, our results proved that LINC01410 regulated HK2 expression by sponging miR-545-3p in NB cells.

\section{Downregulation of LINCOI4I0 Inhibited Tumor Growth and Increased Radiosensitivity by Regulating miR-545-3p and $\mathrm{HK} 2$ in vivo}

To explore the effect of LINC01410 on tumor growth and radiation response in vivo, we established a xenograft model in which the SK-N-BE(2) cells stably transfected with shLINC01410 or sh-NC were subcutaneously injected into $\mathrm{BALB} / \mathrm{c}$ nude mice and irradiated with 6Gy X-ray once a week or did not irradiate. In line with in vitro results, LINC01410 knockdown or IR treatment suppressed tumor volume and weight, and combination of LINC01410 interference and IR treatment significantly inhibited tumor volume and weight in xenograft model compared with only sh-LINC01410 group or IR group (Figure 7A and B). In addition, knockdown of LINC01410 decreased the expression of LINC01410 and HK2 

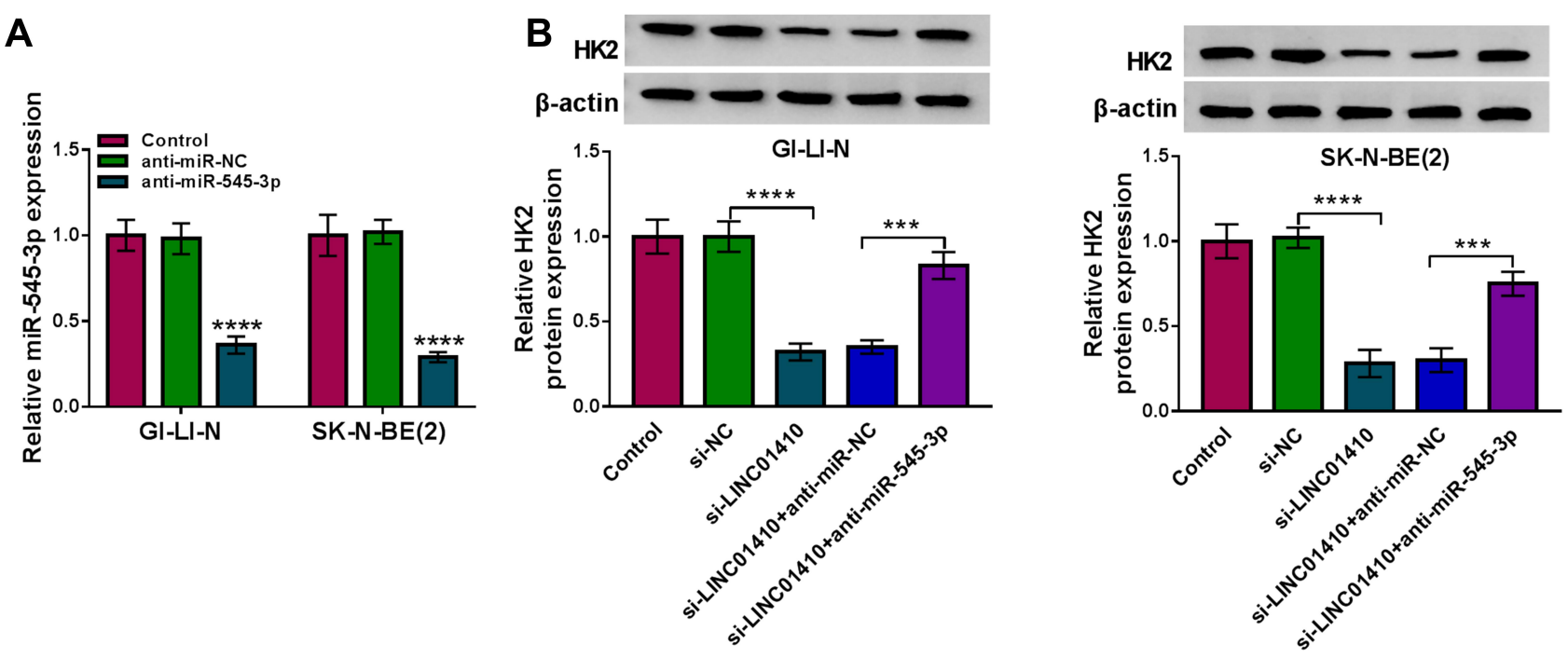

Figure 6 LINC0 1410 positively regulated HK2 expression via sponging miR-545-3p. (A) Knockdown efficiency of miR-545-3p was determined by qRT-PCR. (B) Weston bolt assay was conducted to measure the protein expression of HK2 in GI-LI-N and SK-N-BE(2) cells transfected with si-NC, si-LINCOI4I0, si-LINCOI4IO + anti-miR-NC, or siLINCOI $410+$ anti-miR-545-3p. $* * * P<0.001$, $* * * * P<0.0001$.

while increased the expression of miR-545-3p in excised tumor masses (Figure 7C and D). IHC analysis showed that LINC01410 deficiency reduced HK2-positive cells (Figure 7E), suggesting that LINC01410 knockdown decreased the expression HK2 in tumor tissues. These above findings indicated that downregulation of LINC01410 could enhance the radiosensitivity to inhibit tumor growth in vivo.

\section{Discussion}

NB is considered to be a common and aggressive malignancy in children. Recently, it has been increasingly recognized that dysregulation of lncRNAs is involved in the progression of NB. ${ }^{23,26}$ Radiotherapy has become an effective strategy for NB treatment, however, the influence of lncRNAs on radiosensitivity of NB cells is not well elucidated. The purpose of this research was to explore the effects of LINC01410 on NB progression and radiosensitivity.

Up to now, a growing number of researches have shown that LINC01410 serves as an oncogene and plays essential roles in cellular behaviors, such as cell growth, cell cycle, metastasis and apoptosis. ${ }^{27}$ Besides, Lu et al pointed out that LINC01410 was overexpressed in endometrial cancer tissues, and accelerated endometrial cancer cell progression through miR-23c/CHD7 axis. $^{28}$ Moreover, Jiang et al uncovered that LINC01410 knockdown inhibited cholangiocarcinoma cell proliferation and metastasis via sponging miR-124-3p to decrease SMAD5 expression. ${ }^{29}$ In our research, it was found that LINC01410 abundance was elevated in NB tissues and cells. Additionally, interference of LINC01410 suppressed cell proliferation and invasion, and elevated radiosensitivity via suppressing glycolysis in NB cells as well as restrained tumor growth through enhancing radiosensitivity in vivo. In agreement with our findings, Mi et al disclosed that LINC01410 expression was markedly enhanced in NB tissues and its knockdown inhibited the cell growth and tumor growth and facilitated the apoptosis in NB cells by regulating miR-506-3p/ WEE1 axis. ${ }^{12}$ These results revealed that LINC01410 was involved in the tumorigenesis of NB and radiosensitivity.

Precious research has shown that miR-545-3p has pivotal roles in the regulating cellular behaviors, such as proliferation, differentiation and migration. ${ }^{30,31}$ It has recently been identified as a cancer-related miRNA for several cancers. For example, Changjun et al declared that miR-545-3p was highly expressed in hepatocellular carcinoma tissues and cells, and miR-545-3p inhibition limited hepatocellular carcinoma cell proliferation and metastasis via regulating MT1M. ${ }^{18}$ In contrast, Shi et al proved that miR-545-3p acted as a tumorsuppressive miRNA through targeting HDAC4 to suppress epithelial ovarian cancer proliferation, migration and invasion. $^{32}$ These findings suggested that miR-545-3p could exert a tumor-suppressive or tumor-promotive function depending on the type of cancer. More importantly, Chen et al showed that miR-545 was a downregulated miRNA in $\mathrm{NB},{ }^{20}$ suggesting that miR-545-3p might act as a tumor suppressor in NB. Here, we found that miR-545-3p abundance was declined in NB tissues and cells, and was inversely correlated with LINC01410. Previous studies have 
A

B

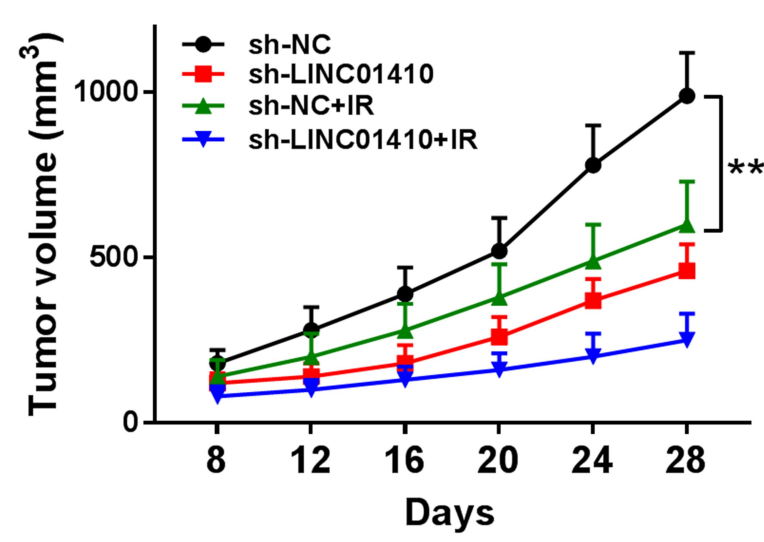

C

D
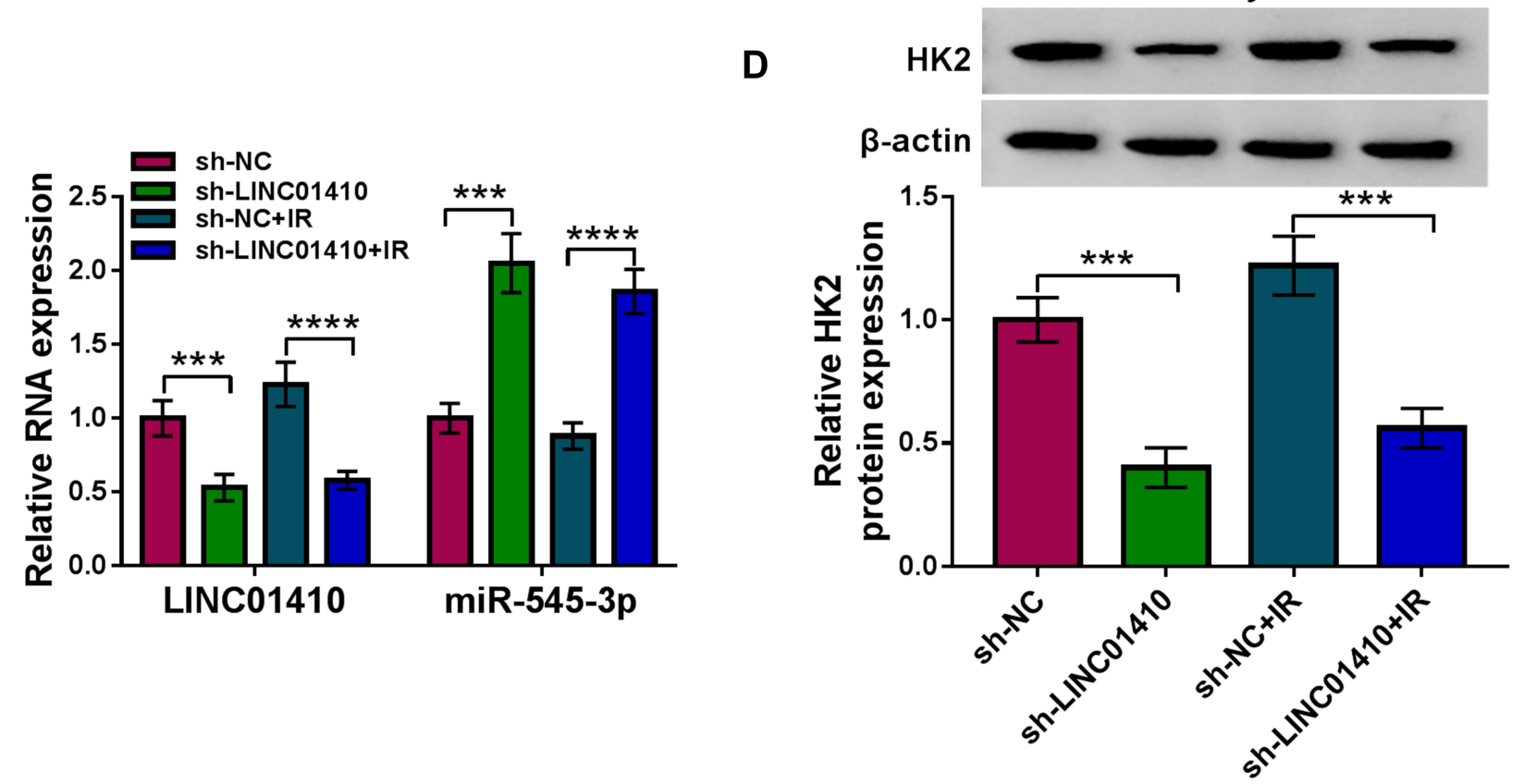

$\mathbf{E}$

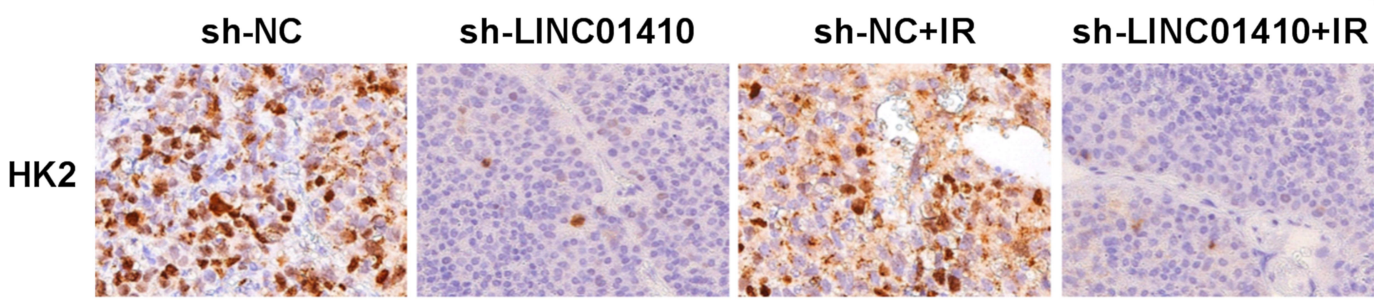

Figure 7 Interference of LINC0 I 110 treatment of IR inhibited tumor growth by upregulating miR-545-3p and downregulating HK2 in vivo. SK-N-BE(2) cells transfected with sh-NC or sh-LINCOI4I0 were injected subcutaneously into nude mice, and the mice were treated with IR or without IR. (A and B) Tumor volume and weight were detected. (C) The expression levels of LINC0I4I0 and miR-545-3p were analyzed by qRT-PCR in resected tumor tissues. (D) Western blot assay was used to measure the protein expression of HK2 in resected tumor tissues. (E) The expression level of HK2 in resected tumor tissues was detected by IHC analysis $* P<0.05$, $* * P<0.0 \mathrm{I}$, $* * * P<0.001$, $* * * * P<0.0001$.

demonstrated that IncRNAs can execute their functions through binding with their downstream miRNAs. ${ }^{33}$ To further explore the relationship between LINC01410 and miR-545-
$3 \mathrm{p}$, starBase v2.0 was used to predict the targeting relationship. Interestingly, starBase v2.0 showed that miR-545-3p contained predicted binding sites with LINC01410. Next, the 
prediction was validated through dual-luciferase reporter, RIP and RNA pull-down assays. In addition, rescue experiments demonstrated that miR-545-3p knockdown could reverse the impact of LINC01410 interference on cell proliferation, invasion, radiosensitivity, and glycolysis in NB cells. Collectively, our data indicated that LINC01410 exerted its function through regulating miR-545-3p expression.

It is well known that the biological functions of miRNAs are realized via modulating mRNA expression, so the potential target genes for miR-545-3p would be analyzed in further analysis. Despite the fact that numerous tumor-associated genes were predicted using starBase v2.0, HK2 was selected as the candidate target gene of miR-545-3p due to its tumorpromoting effect. Further, we proved that miR-545-3p directly targeted HK2. HK2 (a major type of hexokinase family) was reported to be overexpressed and facilitated rates of glucose metabolism necessary for tumor growth in multiple cancers. ${ }^{34,35}$ In addition, Botzer et al presented that high expression of HK2 promoted NB metastasis. ${ }^{36}$ Besides, Cen et al revealed that HK2 was upregulated in NB tissues and cells, and HK2 accumulation weakened the repressive effects of miR-143-3p restoration on progression of $\mathrm{NB}^{23} \mathrm{In}$ line with previous findings, we also demonstrated that HK2 was highly expressed in NB tissues and cells. Moreover, HK2 upregulation could weaken the influence of miR-545$3 p$ restoration on inhibition of tumorigenesis and promotion of radiosensitivity in NB cells. Furthermore, we uncovered that LINC01410 acted as a molecular sponge of miR-545-3p to modulate HK2 expression. In a word, these findings disclosed that LINC01410 regulated the progression and radiosensitivity of NB cells by sponging miR-545-3p to modulate HK2 expression.

In conclusion, LINC01410 and HK2 were upregulated while miR-545-3p was downregulated in NB tissues and cells. Knockdown of LINC01410 inhibited the tumorigenesis and enhanced the radiosensitivity in NB cells by regulating miR-545-3p and HK2 expression. These findings might provide a potential therapeutic strategy for NB.

\section{Acknowledgment}

Thanks for all participants involved in this study.

\section{Disclosure}

The authors declare that they have no competing interests.

\section{References}

1. Matthay KK. Neuroblastoma: biology and therapy. Oncology. 1997;11(12):1857.

2. Ward E, DeSantis C, Robbins A, Kohler B, Jemal A. Childhood and adolescent cancer statistics, 2014. CA Cancer J Clin. 2014;64 (2):83-103. doi:10.3322/caac. 21219

3. Harrison J, Myers M, Rowen M, Vermund H. Results of combination chemotherapy, surgery, and radiotherapy in children with neuroblastoma. Cancer. 1974;34(3):485-490. doi:10.1002/10970142(197409)34:3<485::AID-CNCR2820340302>3.0.CO;2-8

4. Madhusoodhanan R, Natarajan M, Veeraraghavan J, et al. NFкB signaling related molecular alterations in human neuroblastoma cells after fractionated irradiation. J Radiat Res (Tokyo). 2009;50 (4):311-324. doi:10.1269/jrr.08110

5. Wilusz JE, Sunwoo H, Spector DL. Long noncoding RNAs: functional surprises from the RNA world. Genes Dev. 2009;23 (13):1494-1504. doi:10.1101/gad.1800909

6. Prensner JR, Chinnaiyan AM. The emergence of IncRNAs in cancer biology. Cancer Discov. 2011;1(5):391-407. doi:10.1158/2159-8290. CD-11-0209

7. Pandey GK, Mitra S, Subhash S, et al. The risk-associated long noncoding RNA NBAT-1 controls neuroblastoma progression by regulating cell proliferation and neuronal differentiation. Cancer Cell. 2014;26(5):722-737. doi:10.1016/j.ccell.2014.09.014

8. Pan J, Zhang D, Zhang J, Qin P, Wang J. LncRNA RMRP silence curbs neonatal neuroblastoma progression by regulating microRNA-206/tachykinin-1 receptor axis via inactivating extracellular signal-regulated kinases. Cancer Biol Ther. 2019;20 (5):653-665. doi:10.1080/15384047.2018.1550568

9. Wang G, Wang X, Jin Y. LINC01410/miR-3619-5p/FOXM1 feedback loop regulates papillary thyroid carcinoma cell proliferation and apoptosis. Cancer Biother Radiopharm. 2019;34(9):572-580. doi:10.1089/cbr.2019.2854

10. Liu F, Wen C. LINC01410 knockdown suppresses cervical cancer growth and invasion via Targeting miR-2467-3p/VOPP1 axis. Cancer Manag Res. 2020;12:855-861. doi:10.2147/CMAR.S236832

11. Zhang JX, Chen ZH, Chen DL, et al. LINC01410-miR-532-NCF2-NF$\mathrm{kB}$ feedback loop promotes gastric cancer angiogenesis and metastasis. Oncogene. 2018;37(20):2660-2675. doi:10.1038/s41388-018-0162-y

12. Mi J, Han Y, Zhang J, Hao X, Xing M, Shang C. Long noncoding RNA LINC01410 promotes the tumorigenesis of neuroblastoma cells by sponging microRNA-506-3p and modulating WEE1. Cancer Med. 2020;9(21):8133-8143. doi:10.1002/cam4.3398

13. Bach DH, Lee SK, Sood AK. Circular RNAs in Cancer. Mol Ther Nucleic Acids. 2019;16:118-129. doi:10.1016/j.omtn.2019.02.005

14. Ardekani AM, Naeini MM. The role of microRNAs in human diseases. Avicenna J Med Biotechnol. 2010;2(4):161

15. Jansson MD, Lund AH. MicroRNA and cancer. Mol Oncol. 2012;6 (6):590-610.

16. Dickey JS, Zemp FJ, Martin OA, Kovalchuk O. The role of miRNA in the direct and indirect effects of ionizing radiation. Radiat Environ Biophys. 2011;50(4):491. doi:10.1007/s00411-011-0386-5

17. Reddy KB. MicroRNA (miRNA) in cancer. Cancer Cell Int. 2015;15 (1):38. doi:10.1186/s12935-015-0185-1

18. Changjun L, Feizhou H, Dezhen P, Zhao L, Xianhai M. MiR-545-3p/ MT1M axis regulates cell proliferation, invasion and migration in hepatocellular carcinoma. Biomed Pharmacother. 2018;108:347-354. doi:10.1016/j.biopha.2018.09.009

19. Cui J, Pan G, He Q, Yin L, Guo R, Bi H. MicroRNA-545 targets ZEB2 to inhibit the development of non-small cell lung cancer by inactivating Wnt/ $\beta$-catenin pathway. Oncol Lett. 2019;18 (3):2931-2938. doi:10.3892/ol.2019.10619 
20. Chen B, Hua Z, Qin X, Li Z. Integrated microarray to identify the hub miRNAs and constructed miRNA-mRNA network in neuroblastoma via bioinformatics analysis. Neurochem Res. 2020. doi:10.1007/ s11064-020-03155-3

21. Yoshino H, Enokida H, Itesako $\mathrm{T}$, et al. Tumor-suppressive micro RNA-143/145 cluster targets hexokinase-2 in renal cell carcinoma. Cancer Sci. 2013;104(12):1567-1574. doi:10.1111/cas. 12280

22. Fang R, Xiao T, Fang Z, et al. MicroRNA-143 (miR-143) regulates cancer glycolysis via targeting hexokinase 2 gene. J Biol Chem. 2012;287(27):23227-23235. doi:10.1074/jbc.M112.373084

23. Cen Y, Xu L, Huang S, Zhuang S. miR-143-3p functions as a tumor suppressor by targeting HK2 in neuroblastomas. Int J Clin Exp Med. 2019;12(7):8450-8460.

24. Zhou S, Ye W, Ren J, et al. MicroRNA-381 increases radiosensitivity in esophageal squamous cell carcinoma. Am J Cancer Res. 2015;5 (1):267.

25. Hsu PP, Sabatini DM. Cancer cell metabolism: warburg and beyond. Cell. 2008;134(5):703-707. doi:10.1016/j.cell.2008.08.021

26. Bevilacqua V, Gioia U, Di Carlo V, et al. Identification of linc-NeD125, a novel long non coding RNA that hosts miR-125b-1 and negatively controls proliferation of human neuroblastoma cells. RNA Biol. 2015;12(12):1323-1337. doi:10.1080/15476286.2015. 1096488

27. Luo J, Guo Y, Liu X, Yang X, Xiao F, Zhou M. Long non-coding RNA LINC01410 promotes colon cancer cell proliferation and invasion by inhibiting miR-3128. Exp Ther Med. 2018;16(6):4824-4830. doi:10.3892/etm.2018.6806

28. Lu M, Ding N, Zhuang S, Li Y. LINC01410/miR-23c/CHD7 functions as a ceRNA network to affect the prognosis of patients with endometrial cancer and strengthen the malignant properties of endometrial cancer cells. Mol Cell Biochem. 2020;469(1-2):9-19.

29. Jiang T, Wang C, Zhu Y, Han H. LINC01410 promotes cell proliferation and migration of cholangiocarcinoma through modulating miR-124-3p/SMAD5 axis. J Gene Med. 2020;22(6):e3162. doi:10.10 02/jgm.3162
30. Hao R, Wang B, Wang H, Huo Y, Lu Y. lncRNA TUG1 promotes proliferation and differentiation of osteoblasts by regulating the miR-545-3p/CNR2 axis. Braz J Med Biol Res. 2020;53(11):e9798. doi:10.1590/1414-431x20209798

31. Zhong Y, Wang Y, Dang H, Wu X. LncRNA AFAP1-AS1 contributes to the progression of endometrial carcinoma by regulating miR-5453p/VEGFA pathway. Mol Cell Probes. 2020;53:101606. doi:10.1016/ j.mcp. 2020.101606

32. Shi J, Xu X, Zhang D, et al. Long non-coding RNA PTPRG-AS1 promotes cell tumorigenicity in epithelial ovarian cancer by decoying microRNA-545-3p and consequently enhancing HDAC4 expression. J Ovarian Res. 2020;13(1):127. doi:10.1186/s13048020-00723-7

33. Jin B, Jin $\mathrm{H}, \mathrm{Wu} \mathrm{HB}, \mathrm{Xu} J J$, Li B. Long non-coding RNA SNHG15 promotes CDK14 expression via miR-486 to accelerate non-small cell lung cancer cells progression and metastasis. J Cell Physiol. 2018;233(9):7164-7172. doi:10.1002/jcp.26543

34. He H-C, Bi X-C, Zheng Z-W, et al. Real-time quantitative RT-PCR assessment of PIM-1 and hK2 mRNA expression in benign prostate hyperplasia and prostate cancer. Med Oncol. 2009;26(3):303-308. doi:10.1007/s12032-008-9120-9

35. Mathupala S, Ko YH, Pedersen PL. Hexokinase II: cancer's doubleedged sword acting as both facilitator and gatekeeper of malignancy when bound to mitochondria. Oncogene. 2006;25(34):4777. doi:10.1038/sj.onc.1209603

36. Botzer LE, Maman S, Sagi-Assif O, et al. Hexokinase 2 is a determinant of neuroblastoma metastasis. Br J Cancer. 2016;114 (7):759. doi: $10.1038 /$ bjc. 2016.26

\section{Publish your work in this journal}

OncoTargets and Therapy is an international, peer-reviewed, open access journal focusing on the pathological basis of all cancers, potential targets for therapy and treatment protocols employed to improve the management of cancer patients. The journal also focuses on the impact of management programs and new therapeutic agents and protocols on patient perspectives such as quality of life, adherence and satisfaction. The manuscript management system is completely online and includes a very quick and fair peer-review system, which is all easy to use. Visit http://www.dovepress.com/ testimonials.php to read real quotes from published authors. 